\title{
Adaptive Multiple-Model Control of A Class of Nonlinear Systems
}

\author{
Chao Yang \\ The Seventh Research Division and the Department of Systems and Control, Beihang University (BUAA), \\ Beijing 100191, China \\ Yingmin Jia \\ The Seventh Research Division and the Department of Systems and Control, Beihang University (BUAA), \\ Beijing 100191, China \\ E-mails: yangchao_buaa@163.com;ymjia@buaa.edu.cn
}

\begin{abstract}
In this paper, an adaptive multiple-model controller is developed for nonlinear systems in parametric-strict-feedback form. Unlike the previous results, a switching scheme is not required here to switch the most appropriate model into the controller design. The new scheme reduces the number of identification models and uses information provided by all the models more efficiently than previous results by using the convex combination of estimates of parameters. The method guarantees parameter convergence and global asymptotic stability of the closed-loop system. The global boundness of closed-loop signals and asymptotic convergence to zero of tracking error are proved. A simulation example is included to demonstrate the effectiveness of the obtained results.
\end{abstract}

Keywords: adaptive control, multiple-model design, nonlinear systems, asymptotic tracking

\section{Introduction}

In recent years, adaptive control has been well investigated $^{1-5}$. It is proposed to cope with the problem of parametric uncertainties in systems lager than traditional method can handle. The typical idea of adaptive control is estimating unknown parameters online and designing controller with the estimates as the real ones ${ }^{2}$. Plenty of different stable adaptive controllers have been designed for linear or nonlinear uncertain systems $^{7-9}$. A distinguished problem for adaptive control is that transient performance of the system may include unacceptable large peaks, especially when there exist large initial estimation errors. To overcome this drawback and enhance the transient performance, adaptive control using multiple models for linear systems was proposed in Refs.7, 8, 10and 11. The basic idea of Ref.10 was running in parallel multiple models, and design an index of performance based on identification errors, so that model closest to the real plant can be selected by switching quickly and transient response can be improved. Latter, the method was extended to switching and tuning in Refs.12 and 13. Numerous of simulations and applications have demonstrated adaptive multiple-model control can enhance transient performance significantly.

However, the methods are mainly concentrated on linear systems. Adaptive multiple-model control for nonlinear systems is initially considered in Ref.14. Then, adaptive multiple-model control method for nonlinear requiring persistence of excitation is proposed in Ref.15, where the unknown parameters can be calculated in the first place.

In Ref.16, a sufficient condition concerning the parameter convergence is given by constructing a global, explicit strong Lyapunov function. Inspired by this, we develop a novel multiple model adaptive controller for a class of nonlinear system in parametric-strict-feedback form. Unlike the usual hypothesis, the restrictive matching conditions are not required, but the persistency of excitation condition is assumed. The control scheme employs adaptive identification models based on adaptive parameters from a known compact parameter set and a virtual identification model, of which the estimation parameter is both adaptive and resettable. The algorithm reduces the number of identification models by using convex combination method to estimate parameter even as the system is in 
operation. Also, global asymptotic stability of the closed-loop system is proved.

This paper is organized as follows. The problem formulation and assumptions are given in Section 2. In Section 3, backstepping adaptive method and the new adaptive multiple-model method are introduced. In Section 4, the closed-loop system dynamics is analyzed including stability and parameter convergence. Section 5 presents a simulation which compares the proposed method with single model adaptive control.

\section{Problem formulation}

Consider the following nonlinear systems in parametric-strict-feedback form ${ }^{[2]}$ :

$$
\begin{aligned}
\dot{x}_{1} & =x_{2}+f_{1}^{T}\left(x_{1}\right) \boldsymbol{\theta} \\
& \vdots \\
\dot{x}_{\mathrm{i}} & =x_{\mathrm{i}+1}+f_{\mathrm{i}}^{T}\left(x_{1}, x_{2}, \ldots, x_{\mathrm{i}}\right) \boldsymbol{\theta}, \quad i=1,2 \ldots n-1 \\
\dot{x}_{n} & =u+f_{n}^{T}\left(\bar{x}_{n}\right) \boldsymbol{\theta} \\
y & =x_{1}
\end{aligned}
$$

Where $x_{i} \in R, i=1,2 \ldots n$ is the state of system, $u \in R$ is the control input, $\boldsymbol{\theta} \in R^{p}$ is a $\mathrm{n}$ unknown parameter vector belonging to a known compact set $S \in R^{p}$ and $y \in R$ is the output. The functions

$f_{i}, i=1,2, \cdots, i$ are known smooth functions. $y_{r} \in R$ is the reference signal to be tracked. We suppose th at the full system states are available. The object is to improve transient performance in the presence of large parametric uncertainties, and meanwhile, ensure th e stability of the closed-loop system.

It should be noted that in the classical backstepping adaptive control design, transient performance can be improved by choosing sufficiently large high-gain parameters ${ }^{2}$. However, a possible shortcoming of such method is that the resulting control efforts may be very large. Another way to improve transient performance is using multiple identifications models.

Before the design of adaptive multiple-model controller, we need necessary assumptions which are useful in the following analysis.

Assumption 1(A1) There is a known positive realvalued constant B satisfying

$\max \left\{\left|y_{r}(t)\right|_{\infty},\left|\dot{y}_{r}(t)\right|_{\infty}\right\} \leq B$

Assumption 2(A2) $f_{1}\left(y_{r}(t)\right)$ satisfies the classical persistent excitation condition, that is, for all $t \in R$, there exist positive real-valued constants $\mu$ and $T$ such that

$$
\mu I \leq \int_{t-T}^{t} f_{1}\left(y_{r}(l)\right) \square f_{1}\left(y_{r}(l)\right)^{T} \mathrm{~d} l
$$

\section{Design of adaptive multiple model controller}

\subsection{Introduction of backstepping adaptive control ${ }^{[2]}$}

The standard backstepping adaptive control and adaptation law for parametric-strict-feedback system are given by

$u=\alpha_{n}\left(x, \hat{\theta}, \bar{y}_{r}^{(n)}\right)$
$\dot{\hat{\theta}}=\Gamma \tau_{n}=\Gamma W Z$

where

$$
\begin{aligned}
\boldsymbol{\theta}= & {\left[\theta_{1}, \theta_{2}, \cdots, \theta_{p}\right]^{T}, \tilde{\boldsymbol{\theta}}=\hat{\boldsymbol{\theta}}-\boldsymbol{\theta} } \\
Z= & {\left[z_{1}, z_{2}, \cdots, z_{n}\right]^{T} } \\
z_{1}= & y-y_{r}, z_{i}=x_{i}-\alpha_{i-1}, 2 \leq i \leq n, \\
W= & {\left[\omega_{1}, \omega_{2}, \cdots, \omega_{n}\right] } \\
\omega_{1}= & f_{1}, \omega_{i}=f_{i}-\sum_{k=1}^{i-1} \frac{\partial \alpha_{i-1}}{\partial x_{k}} f_{k}, \\
\tau_{1}= & \omega_{1} z_{1}, \tau_{i}=\tau_{i-1}+\omega_{i} z_{i}, \\
\alpha_{1}=- & -c_{1} z_{1}-\hat{\theta}^{T} f_{1}\left(x_{1}\right)+\dot{y}_{r}(t), \\
\alpha_{i}= & -z_{i}-c_{i} z_{i}-\hat{\theta} \omega_{i}+\frac{\partial \alpha_{i-1}}{\partial \hat{\theta}} \Gamma \tau_{i} \\
& +\sum_{k=1}^{i-1}\left(\frac{\partial \alpha_{i-1}}{\partial x_{k}} x_{k+1}+\frac{\partial \alpha_{i-1}}{\partial x_{k}} x_{k+1}+\frac{\partial \alpha_{i-1}}{\partial y_{r}^{(k-1)}} y_{r}^{(k)}\right) \\
& \quad+\sum_{k=2}^{i-1}\left(\frac{\partial \alpha_{k-1}}{\partial \hat{\theta}}\right) \Gamma \omega_{i} z_{k}+\dot{y}_{r}(t), 2 \leq i \leq n
\end{aligned}
$$

where $\Gamma=\Gamma^{T}>0$ is the adaptation gain matrix, $y_{r}$ is the reference signal to be tracked and $c_{i}>0,1 \leq i \leq n$ are constants to be designed. The above control design is based on the Lyapunov function $V_{n}\left(z_{1}, z_{2}, \cdots, z_{n}, \bar{\theta}\right)=\frac{1}{2} \sum_{i=1}^{n} z_{i}^{2}+\frac{1}{2}(\hat{\theta}-\theta)^{T} \Gamma^{-1}(\hat{\theta}-\theta)$

The time derivative of $V_{n}$, computed with (5)-(10) is $\dot{V}_{n}\left(z_{1}, z_{2}, \cdots, z_{n}, \bar{\theta}\right) \leq-\sum_{i=1}^{n} c_{i} z_{i}^{2} \quad$.This implies the boundness of the state $z_{i}, 1 \leq i \leq n$ and $\hat{\theta}$, which in turn indicates the boundness of system state $x_{i}, 1 \leq i \leq n$ and control $u$. Under the standard arguments of adaptive control theory, it follows that $z_{i}(t), 1 \leq i \leq n$ tend to zero asymptotically, and thus $\lim _{t \rightarrow \infty} z_{1}(t)=\lim _{t \rightarrow \infty}\left(y(t)-y_{r}(t)\right)=0$. Then asymptotic tracking can be achieved.

Remark: In addition to the boundness of the parameter estimation, there are seldom results about parameter convergence. Ref.6 provides sufficient condition for parameter estimates convergence, but the condition is difficult to verify. With assumptions (2) and (3), Ref.16 constructs an explicit, global, strong Published by Atlantis Press

Copyright: the authors 
Lyapunov function, and proves parameter estimation convergence. See Ref.16 for more details.

\subsection{Design of adaptive multiple-model controller}

We will run in parallel $(\mathrm{p}+2)$ models. $\mathrm{N}(\mathrm{N}=\mathrm{p}+1)$ models are in the same structure but different initial parameter values $\theta_{j}(0), j=1,2 \ldots, N$. The initial values of $\theta_{j}(0), j=1,2 \ldots, N$ are known and the region of uncertainty $\mathrm{S}$ of the plant parameter vector $\boldsymbol{\theta}$ lies in their convex hull $\mathrm{K}$ (i.e. $\mathrm{S} \subset \mathrm{K}$ ). The other one model called

' virtual model' is designed with adaptive and resetting mechanism. The $\mathrm{N}$ models will run parallel and parameter estimates are evaluated online by a criterion function $J(t)$.Define

$$
J_{j}(t)=\alpha e_{j}^{2}(t)+\beta \int_{0}^{t} e^{t-\tau} e_{j}^{2}(\tau) d \tau
$$

as the criterion function for the $j$-th adaptive model, where $\alpha>0, \beta>0$ are constants to be designed. $\mathrm{J}_{\mathrm{c}}$ is the criterion function for virtual model. At each resetting time $t_{r}$, if $J_{c}>\gamma \cdot \min \left\{J_{1}, \cdots, J_{N}\right\}$, where $\gamma \in(0,1)$ is a constant to be designed, reset the estimate parameter vector $\theta_{e}(t)$ of virtual model by

$$
\theta_{e}(t)=\sum_{i=1}^{N} \gamma_{i} \theta_{i}
$$

where

$$
\gamma_{i}=\frac{1}{J_{i}(t)} / \sum_{j=1}^{N} \frac{1}{J_{j}(t)}, i=1,2, \cdots, \mathrm{N} \cdot
$$

Otherwise, the estimate parameters remain unchanged and the process repeats. An obvious problem is that whether the resetting is finite or infinite. In the following section, we will prove that only a finite number of resetting can occur.

\section{Stability analysis}

Theorem Suppose assumptions (2),(3) hold, and the adaptive multiple-model controller (4) and adaption law (5) in the paper are applied to system (1). Then, for all initial conditions only a finite number of resetting can occur, all closed-loop states and $\theta_{j}, j=1,2, \cdots, n$ are bounded, furthermore asymptotic tracking is achieved ,i.e., $\lim _{t \rightarrow \infty}\left(y(t)-y_{r}(t)\right)=0$ as $t \rightarrow \infty$.

Proof. First, we will prove that the number of resetting is finite. After the above analysis, we have $\quad \overline{\theta_{j}}(t) \in L_{\infty}[0, \infty), j=1,2, \cdots, n$, for $i=1,2, \cdots, n, \forall \grave{\mathrm{o}}>0, \exists t_{i} \in[0, \infty)$, such that $\left|\bar{\theta}_{i}(t)\right|<\grave{\mathrm{o}}, \forall t>t_{i}$. Let $\quad \mathrm{t}_{\max }=\max \left\{\mathrm{t}_{1}, \mathrm{t}_{2}, \ldots, \mathrm{t}_{\mathrm{n}}\right\} . \quad$ For $t>t_{\max },\left|\bar{\theta}_{i}(t)\right|<\mathrm{o}, i=1,2, \cdots, \mathrm{N}$. Based on the resetting mechanism, $\left|\bar{\theta}_{e}(t)\right|=\left|\sum_{j=1}^{n} \gamma_{j} \bar{\theta}_{j}(t)\right| \leq \max \left\{\left|\bar{\theta}_{j}(t)\right|, j=1,2, \ldots, \mathrm{N}\right\}<\dot{0} . \quad$ Then the resetting adaptive model will estimate parameters with zero error. So, in the worst situation, the amount of resetting $N_{r}=\left[\frac{t_{\max }}{T}\right]$, where $T$ is the resetting interval, and [·] means the floor function. Thus finite number of resetting is proved. Further, after every resetting time $t_{r}$, all adaptive models and the resetting model operate under respective control input and adaptation law, then all closed-loop state is bounded. Once $t>t_{\max }$, the system will operate under the classical backstepping scheme, so we can further point out that $\lim _{t \rightarrow \infty}\left(y(t)-y_{r}(t)\right)=0$.This completes the whole proof.

\section{Simulation result}

The nonlinear system in parametric-strict-feedback form is as follows:

$$
\begin{aligned}
\dot{x}_{1} & =x_{2}+\theta x_{1} \\
\dot{x}_{2} & =u+\theta \cos \left(x_{1} x_{2}\right) \\
y & =x_{1}
\end{aligned}
$$

where $\theta \in[0.1,5.5]$ is an unknown parameter. The output $y=x_{1}$ is to asymptotically track the reference signal $y_{r}=\sin t$.

In simulation, the controller and adaptation law is developed as (4), (5). For all constant $B>1$, (2) holds.

For any $\mu_{0} \in(0, \pi), \int_{t-2 \pi}^{t} y_{r}^{2}(l) d l \geq \mu_{0}$ follows for all $t \in R$, then let $\mu=\mu_{0}, T=2 \pi$, and (3) holds. The unknown parameter is $\theta=1, c_{1}=1.2, c_{2}=1$, the adaptive gain $\gamma=1$, initial state $x_{1}(0)=x_{2}(0)=0$, and the initial estimates of parameter for classical adaptive control and adaptive multiple-mode control are $\theta_{1}(0)=2.8, \theta_{2}(0)=0.1, \theta_{3}(0)=5.5, \theta(0)=2.8$

As seen from Figure 1, the state $x_{1}$ of the system is bounded, and asymptotic tracking is achieved. Adaptive multiple-model control (d) performs better than single model (a-c) especially when initial parameter estimation error is large (b and c). In Figure 2, all parameter estimations of $\theta$ asymptotically converge to its true value while the estimation in adaptive multiple-model control (d) is the fastest. Figure 3 illustrates the control input $u$ is bounded in the two different control schemes. Thus the scheme proposed in this paper is feasible and effective.
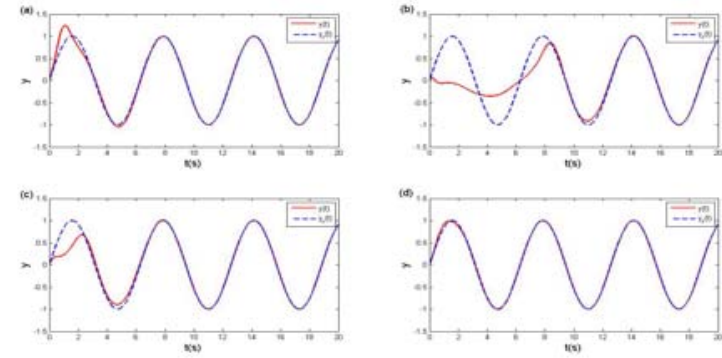

Fig.1. Output and reference signal: (a), (b), (c) single model with $\theta(0)=0.1,5.5,2.8$ respectively, and (d) retting model with $\theta(0)=2.8$

Published by Atlantis Press

Copyright: the authors 


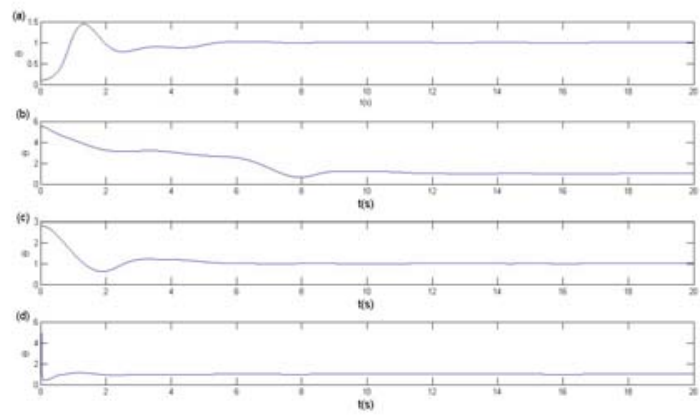

Fig.2. Parameter estimation: (a), (b), (c) single model with $\theta(0)=0.1,5.5,2.8,(\mathrm{~d})$ retting model with $\theta(0)=2.8$

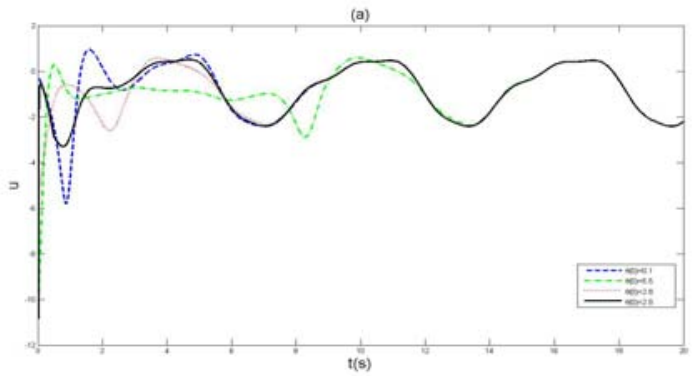

Fig.3. control input $\mathrm{u}$ : dashed line, dash-dot line and dotted line for single model with $\theta(0)=0.1,5.5,2.8$, solid line for virtual model with $\theta(0)=2.8$

\section{Conclusion}

In this paper, an adaptive multiple-model controller is developed for a class of nonlinear systems in parametric-strict-feedback form. Unlike previous results, a switching scheme is not necessary to guarantee the model closest to the real plant to be switched into the controller design. Global asymptotic stability of the closed-loop system, globally uniformly bounded of all the closed-loop signals and convergence of parameter estimates are proved. The simulation results illustrate the feasibility and effectiveness of the proposed method.

\section{Acknowledgements}

This work was supported by the National Basic Research Program of China (973 Program: 2012CB821200, 2012CB821201) and the NSFC (61134005, 60921001, 61327807).

\section{References}

[1] Narendra KS, Annaswamy AM (1989), Stable Adaptive Systems. Prentice Hall Inc.: Englewood Cliffs, NJ

[2] Krstic M, Kanellakopoulos I, Kokotovic PV (1995), Nonlinear and adaptive control design. Wiley, New York [3]Tao G (2003), Adaptive Control Design and Analysis. John Wiley \& Sons
[4] Ioannou PA, Sun J (1996), Robust Adaptive Control. Prentice Hall: Englewood Cliffs, NJ

[5] Middleton RH, Goodwin G C, Hill D J, Mayne D Q (1988), Design issues in adaptive control. IEEE Trans. Automat. Control 33:50-58.

[6] Lin JS, Kanellakopoulos I (1999) Nonlinear enhance parameter convergence in strict feedback systems. IEEE Trans. Automat. Control 44:89-94

[7] Morse AS (1996), Supervisory control of families of linear set-point controller, part 1: Exact matching, IEEE Trans. Automat. Control 41:1413-1431.

[8] Anderson BDO, Brinsmead T S, Bruyne FD, et al. (2000), Multiple model adaptive control, part 1: Finite controller coverings. Int. J. Robust Nonlinear Control 10:909-929

[9] Morse AS (1997), Supervisory control of families of linear set-point controller. 2. Robustness. IEEE Trans. Automat. Control 42:1500-1515

[10]Narendra KS, Balakrishnan J (1994), Improving transient response of adaptive control systems using multiple models and switchings. IEEE Trans. Automat. Control 39:1861-1866

[11]Narendra KS, Balakrishnan J (1997), Adaptive control using multiple models. IEEE Trans. Automat. Control 42:71-187

[12]Han Z, Narendra K S (2010), Multiple adaptive models for control. Proceedings of the 49th IEEE conference on decision and control, Atlanta, Georgia, 60-65

[13]Narendra KS, Han Z (2011). The changing face of adaptive control: the use of multiple models. Annual reviews in control 35:1-12

[14]Narendra KS, George K (2002), Adaptive control of simple nonlinear systems using multiple models. Proceedings of American Control Conference: 17791784

[15]Ciliz MK, Cezayirli A (2006), Increased transient performance for the adaptive control of feedback linearizable systems using multiple models, Int. J. Control 79:205-1215

[16]He B L, Li J M (2011), Systematic method of parameter convergence in strict-feedback nonlinear systems (in Chinese).J.Sys.Sci.Math.Scis.31(5):501-511

Published by Atlantis Press

Copyright: the authors 